OPEN ACCESS

Edited by:

Francesc Xavier Guix, Severo Ochoa Molecular Biology Center (CSIC-UAM), Spain

Reviewed by: YeXiong,

Henry Ford Health System,

United States

Bogdan A. Stoica, University of Maryland, Baltimore,

United States

*Correspondence:

Edward J. Goetzl edward.goetzl@ucsf.edu

Aurélie Ledreux

aurelie.ledreux@du.edu

Dimitrios Kapogiannis

kapogiannisd@nih.gov

Specialty section: This article was submitted to Neurodegeneration, a section of the journal

Frontiers in Neuroscience

Received: 22 February 2019

Accepted: 23 April 2019

Published: 08 May 2019

Citation:

Goetzl EJ, Ledreux A, Granholm A-C, Elahi FM, Goetzl L, Hiramoto J and Kapogiannis D (2019)

Neuron-Derived Exosome Proteins

May Contribute to Progression From

Repetitive Mild Traumatic Brain Injuries to Chronic Traumatic

Encephalopathy.

Front. Neurosci. 13:452.

doi: 10.3389/fnins.2019.00452

\section{Neuron-Derived Exosome Proteins May Contribute to Progression From Repetitive Mild Traumatic Brain Injuries to Chronic Traumatic Encephalopathy}

\author{
Edward J. Goetzl ${ }^{1 *}$, Aurélie Ledreux ${ }^{2 *}$, Ann-Charlotte Granholm², Fanny M. Elahi3, \\ Laura GoetzI ${ }^{4}$, Jade Hiramoto ${ }^{5}$ and Dimitrios Kapogiannis ${ }^{*} *$
}

'Department of Medicine, University of California, San Francisco, San Francisco, CA, United States, ${ }^{2}$ Knoebel Institute for Healthy Aging, University of Denver, Denver, CO, United States, ${ }^{3}$ Department of Neurology, Memory and Aging Center, University of California, San Francisco, San Francisco, CA, United States, ${ }^{4}$ Department of Obstetrics, Gynecology and Reproductive Sciences, Health Sciences Center at Houston, University of Texas, Houston, TX, United States, ${ }^{5}$ Division of Vascular Surgery, Department of Surgery, University of California, San Francisco, San Francisco, CA, United States,

${ }^{6}$ Laboratory of Neurosciences, Intramural Research Program, National Institute on Aging, Baltimore, MD, United States

The recent recognition that Alzheimer disease-like pathology may be found in chronic traumatic encephalopathy (CTE) even after acute mild traumatic brain injury (mTBI) has increased the urgency of elucidating mechanisms, identifying biomarkers predictive of high risk of development of CTE, and establishing biomarker profiles indicative of impactful effects of treatments. Of the many proteins that are loaded into neuron-derived exosomes (NDEs) from damaged neurons after acute TBI, the levels of prion cellular protein (PRPC), coagulation factor XIII (XIIla), synaptogyrin-3, IL-6, and aquaporins remain elevated for months. Prolonged heightened expression of aquaporins and IL-6 may account for the persistent central nervous system edema and inflammation of CTE. PRPc, XIIla and synaptogyrin-3 bind and concentrate neurotoxic forms of oligomeric amyloid $\beta$ peptides or P-tau for delivery into neurons at or distant from the site of trauma. Our progression factor hypothesis of CTE asserts that physiological neuronal proteins, such as PRPc, XIIla, synaptogyrin-3, IL-6 and aquaporins, that increase in concentration in neurons and NDEs for months after acute TBI, are etiological contributors to CTE by either direct actions or by recruiting neurotoxic forms of $A \beta$ peptides or P-tau. Such progression factors also may be useful new targets for development of drugs to prevent CTE.

Keywords: amyloid, cellular prion protein, phospho-tau, synaptogyrin-3, Alzheimer disease

Abbreviations: $A \beta 42, \beta$ amyloid peptide 1-42; APP, amyloid precursor protein; BACE-1, beta-secretase-1 or beta-site amyloid precursor protein cleaving enzyme 1; BBB, blood-brain barrier; CTE, chronic traumatic encephalopathy; DU, University of Denver; MCP, monocyte chemoattractant protein; mTBI, mild TBI; NDE, neuron-derived exosome; NKCC-1, sodium-potassium-chloride co-transporter-1; PRPc, cellular prion protein; Rab 7, Rab 10, Rab 35, ras-related small GTPase 7, 10, 35; TBI, traumatic brain injury; UC, University of California; UCH-L1, ubiquitin C-terminal hydrolase L1; XIIIa, coagulation factor XIII. 


\section{INTRODUCTION}

Traumatic brain injury (TBI) is the term used to describe mechanical injury to the parenchymal tissues and meninges of the brain, and the subsequent series of further damaging proteolytic, oxidative, inflammatory, and proteinopathic responses (Langlois et al., 2006; Roozenbeek et al., 2013; Quillinan et al., 2016). Several million TBIs occur annually in the United States, of which approximately $80 \%$ are defined as mild traumatic brain injury (mTBI) (Faul and Coronado, 2015; Taylor et al., 2017). A high frequency of repetitive mTBIs is observed in young adults from participation in sports and from the shock waves created by military explosives (Broglio et al., 2009; Champion et al., 2009; Wilk et al., 2012; Papa et al., 2015; McKee et al., 2016). It is clear that moderate and severe TBI predispose to both more prevalent and earlier onset Alzheimer disease, and to CTE (Barnes et al., 2014; Gardner et al., 2014; McKee et al., 2016; Perry et al., 2016). Despite the far higher prevalence of mTBI than moderate and severe TBI, it has only recently been recognized that repetitive mTBIs also increase the risk of subsequent development of CTE and dementia (Crane et al., 2016; Barnes et al., 2018). Our understanding of the mechanisms of acute mTBI resulting from a single concussive blow and CTE following repeated episodes of acute mTBI is incomplete, in part due to the past lack of sensitive and accurate biomarkers for the many constituent abnormalities.

The complex matrix of pathways interacting in the course of an mTBI begin with disruption of the blood-brain barrier (BBB) and axonal injury. BBB damage is manifested maximally within hours by morphological changes in endothelial cells, pericytes and astrocytes, increases in the number of perivascular monocytes, and elevated blood levels of tight-junction and astrocyte-specific proteins (Blyth et al., 2009; Higashida et al., 2011; Obermeier et al., 2013; Kuriakose et al., 2018). Subsequent edema of neurons and interstitial tissues follows with increased brain tissue levels of proteins from damaged neuronal framework structures, channels, transporters, and vesicle transport systems (Hue et al., 2016; Kuriakose et al., 2018). Inflammation develops in the same time period of initial responses to acute mTBI, as shown by increased brain tissue levels of cytokines, such as IFN- $\alpha$, IL- $1 \beta$, IL- 6 , and MCP, oxygen radicals and matrix metalloproteinases (Helmy et al., 2011; Woodcock and MorgantiKossmann, 2013; Yan et al., 2014; Gyoneva and Ransohoff, 2015; Lozano et al., 2015; Simon et al., 2017; Roselli et al., 2018). This period also is characterized by increased expression of stress response and pro-apoptotic factor genes (Mettang et al., 2018).

Onset of more persistent features of CTE months or years after a TBI may be foreshadowed by sustained neuronal elevations of elements of proteinopathic neurodegeneration and chronic inflammation (DeKosky et al., 2013). Although neuropathogenic bridges linking acute mTBI to CTE are poorly understood, some mechanisms have been recognized. Within $24 \mathrm{~h}$ of acute TBI in rats, hippocampal and cortical brain tissues showed transient increases in beta-secretase-1 (BACE-1) (Blasko et al., 2004). In humans suffering single-incident severe TBI, increases in brain tissue levels of APP and A $\beta$ peptides as well as diffuse A $\beta$ plaques may be found within hours and persist variably (DeKosky et al., 2013). In contrast, the predominant early neuropathology in CTE following repetitive mTBIs is patchy distribution of neuropil threads and neurofibrillary tangles of P-tau throughout the neocortex (DeKosky et al., 2013). Plasma levels of tau-positive exosomes were abnormally elevated in former professional football players, exposed to repetitive mTBIs, and these increases correlated with decreases in memory and psychomotor speed (Stern et al., 2016). Although many abnormalities of cognition and affect or mood are similar in subjects suffering from CTE after acute severe TBI or repetitive mTBI, relationships between these neurocognitive abnormalities and differing proteinopathic etiologic mechanisms have not been delineated (Prince and Bruhns, 2017; Roy et al., 2017; Dailey et al., 2018).

Investigations of a wide range of neurally derived proteins and of systemic predominantly lipid metabolites in CSF and plasma have not yielded biomarkers that reliably assess the severity of mTBI, predict progression of mTBI to CTE, or offer useful targets for therapy (DeKosky et al., 2013; Agoston et al., 2017; Werhane et al., 2017; Kim et al., 2018). Changes in blood levels of some individual proteins, such as tau, s100B, neurofilament light chain, ubiquitin protein hydrolase-1 and neuron-specific enolase, showed moderate diagnostic and/or prognostic value in some circumstances, but had limited neural specificity, late expression and lack of cross-correlation (Di Battista et al., 2013). A comprehensive gas chromatographic analysis of sera obtained within 2 weeks of mTBI or more severe TBI showed elevations of two medium-chain fatty acids and 2,3-bisphosphoglyceric acid, that were significantly associated with the severity of TBI and added value to clinical and radiological imaging results (Oresic et al., 2016). Six plasma metabolites, including two fatty acids, three phospholipids and tauroursodeoxycholic acid, that were quantified at $<6 \mathrm{~h}$ to 7 days after mTBI, significantly distinguished $\mathrm{mTBI}$ athletes from matched uninjured teammate controls throughout the week after mTBI (Fiandaca et al., 2018). However, as for the prior investigation of metabolomic lipids, these analytes have no known specific CNS functions and statistical characteristics of data for two independent cohorts showed major differences. It thus seems appropriate now to investigate subjects with acute or chronic repetitive mTBI by quantifying alterations in levels of their endosomal and other membrane CNS proteins, that are accessible in the protected cargo of plasma NDEs. The multiphasic and multicomponent set of events characteristic of all TBIs has suggested the importance of selecting biomarkers that probe both intracellular processes and diverse relevant mechanisms.

\section{PLASMA NEURON-DERIVED EXOSOME PROTEIN ABNORMALITIES IN MTBI}

Application of established technology to enrich the NDE subset of extracellular vesicles in plasma has permitted initial elucidation of the changes in exosome trafficking and levels of cargo proteins characteristic of mTBIs. A study of military personnel suffering mTBI and subsequent chronic unspecific CNS symptoms revealed significant increases in CD81 exosome marker-normalized NDE levels of total tau, $A \beta 42$, and IL10 relative to deployed controls without a history of mTBI 
(Gill et al., 2018). Although the precise timing of episodes of mTBI was not determinable, most had been in combat at least once during the year preceding testing. Significant associations were identified between post-concussive symptoms and increases in NDE tau and between symptoms of post-traumatic stress disorder and increases in NDE IL-10 (Gill et al., 2018).

A recent investigation of plasma levels of NDEs and a broader profile of NDE cargo proteins has been conducted in college athletes aged 18-26 years, who provided plasma within 1 week after a sports-related mTBI (acute mTBI, $n=18$ ) or 3 months or longer after two to four mTBIs (chronic mTBI, $n=14$ ) (Goetzl et al., 2019). Plasma levels of NDEs, assessed by closely correlated counts and levels of extracted CD81 exosome marker, were significantly depressed by a mean of $45 \%$ in acute mTBI $(P<0.0001)$, as compared to teammates without TBI $(n=21)$, but not in chronic mTBI. Mean CD81-normalized NDE concentrations of a range of functional brain proteins were significantly abnormal relative to those of uninjured teammate controls in acute but not chronic mTBI groups, including ras-related small GTPase 10 (Rab 10), 73\% decrease; annexin VII, 8.8-fold increase; UCH-L1, 2.5-fold increase; $\alpha$ II spectrin fragments, 1.9-fold increase; claudin-5, 2.7-fold increase; and sodium-potassium-chloride cotransporter-1 (NKCC-1), 2.8-fold increase (all acute increases were significantly different from controls, $P<0.0001$ ). Three physiological neural proteins whose NDE levels changed significantly in acute mTBI also maintained lesser alterations in chronic TBI. Aquaporin 4 showed 8.9-fold $(P<0.0001)$ and 3.6-fold $(P=0.0004)$ increases, and synaptogyrin-3 showed 3.1-fold $(P<0.0001)$ and 1.3-fold $(P=0.041)$ increases, whereas ras-related small GTPase 35 (Rab 35) exhibited 56\% $(P<0.0001)$ and 33\% $(P=0.0045)$ decreases, respectively, in acute and chronic mTBI. In chronic mTBI, there were elevated CD81-normalized NDE levels of usually neuropathologic $\beta$-amyloid peptide $1-42$ (A $\beta 42)$, 1.6-fold ( $P<0.0001$ relative to controls); XIIIa, 1.3 -fold $(P=0.042)$; P-T181-tau, 2.2-fold $(P<0.0001)$; P-S396-tau, 1.6fold $(P<0.01)$; IL-6, 16-fold $(P<0.0001)$; and prion cellular protein $(\mathrm{PRPC}), 5.1$-fold $(P<0.0001)$, with lesser or greater (XIIIa, IL-6, PRPc) increases in acute mTBI.

The acute decreases in Rab 10 and Rab 35 levels in NDEs, that are presumed to reflect neuronal levels of these small GTPases involved in vesicular formation, trafficking and secretion, may account for the observed acute decreases in plasma levels of NDEs, as discussed in the next section (Figure 1). The sustained decrease in NDE level of Rab35 distinguishes this response from the transient acute decrease in NDE level of Rab10. Levels of other NDE cargo proteins, that represent disposal of damaged neuronal proteins, instead significantly increased after acute $\mathrm{mTBI}$ but these increases were not all sustained in chronic mTBI. Of the acute mTBI NDE cargo responders, annexin VII has roles in neuronal survival and membrane transport functions, $\mathrm{UCH}$ L1 targets damaged proteins by ubiquitination for elimination, $\alpha$ II spectrin with actin constitutes the neuronal periodic skeleton, claudin-5 is a component of the tight junction of the BBB, and NKCC-1 and aquaporins, respectively control ion and water movements required for normal neuronal functions (Creutz, 1992; Ohara et al., 1998; Brophy et al., 2011; Scharfman and
Binder, 2013; Morita et al., 2014). In contrast, the NDE proteins that increased in concentration after chronic repetitive mTBIs without (only P-S396-tau) or with significant increases in acute mTBI are regarded predominantly as mediators of proteinopathic neurodegeneration, including total tau, P-T181-tau, P-S396-tau, A 342 , IL-6, PRPc, and XIIIa. Of this group, PRPc and XIIIa, as well as the acute responder synaptogyrin-3 remain elevated through repetitive mTBIs and bind, concentrate and deliver the mediators of proteinopathic neurodegeneration P-tau or $\mathrm{A} \beta 42$ to other neurons.

Hypoxic or ischemic CNS damage in two disease states evoked some of the same changes in plasma levels of NDEs and/or cargo proteins as were observed in mTBI. In the first example, ten patients undergoing multi-branched endovascular repair of aortic aneurysms were studied pre-operatively, immediately postoperatively and the day after surgery. This form of surgery is often associated with ischemic hyperglycemic spinal damage and prolonged lower extremity weakness despite application of intraoperative counter-measures (Hiramoto et al., 2017). The mean level of NDEs fell significantly after surgery and returned the following day to that observed pre-operatively. The NDE mean level of Rab 10 also decreased post-operatively and rose to near normal the next day. NDE mean levels of PRPc, neurofilament light chain, NKCC-1, aquaporins and several synaptic proteins increased significantly after surgery and decreased to near normal the next day. This contrasts with the sustained elevations of PRPc, aquaporins and synaptogyrin-3 after mTBI. NDE levels of differently phosphorylated forms of insulin receptor signaling protein, changed post-operatively in accordance with development of insulin resistance. The mean NDE level of several other proteins, such as IL-6, rose post-operatively, and fell the next day, but remained significantly higher than that seen preoperatively, which again raised the possibility of a subset of neural protein progression factors that might mediate subacute or chronic injury after hypoxia or ischemia.

The second example is neonatal hypoxemic-ischemic encephalopathy and early identification of affected infants that are not responding to standard therapeutic hypothermia. Neonatal NDEs in plasma obtained at 8,10 and $14 \mathrm{~h}$ after initiation of hypothermia were enriched by absorption with antiContactin-2 antibody (Goetzl et al., 2018). CD81-normalized levels of neonatal NDE synaptopodin, synaptophysin, neuronspecific enolase and mitochondrial cytochrome $\mathrm{C}$ oxidase were quantified immunochemically. The most promising marker for all clinical outcomes was NDE synaptopodin, as the slope of change in its NDE level between 8 and $14 \mathrm{~h}$ of hypothermia was associated with clinically significant outcomes including persistent seizure activity requiring treatment $(p=0.02)$ and length of hospital stay $(P<0.001)$.

\section{Rab SMALL GTPases AND NDE TRAFFICKING in mTBIs}

Rab small GTPases, the largest branch of the Ras superfamily, are highly abundant, and diverse organizers of intracellular membrane trafficking in the CNS (Kiral et al., 2018). The state 


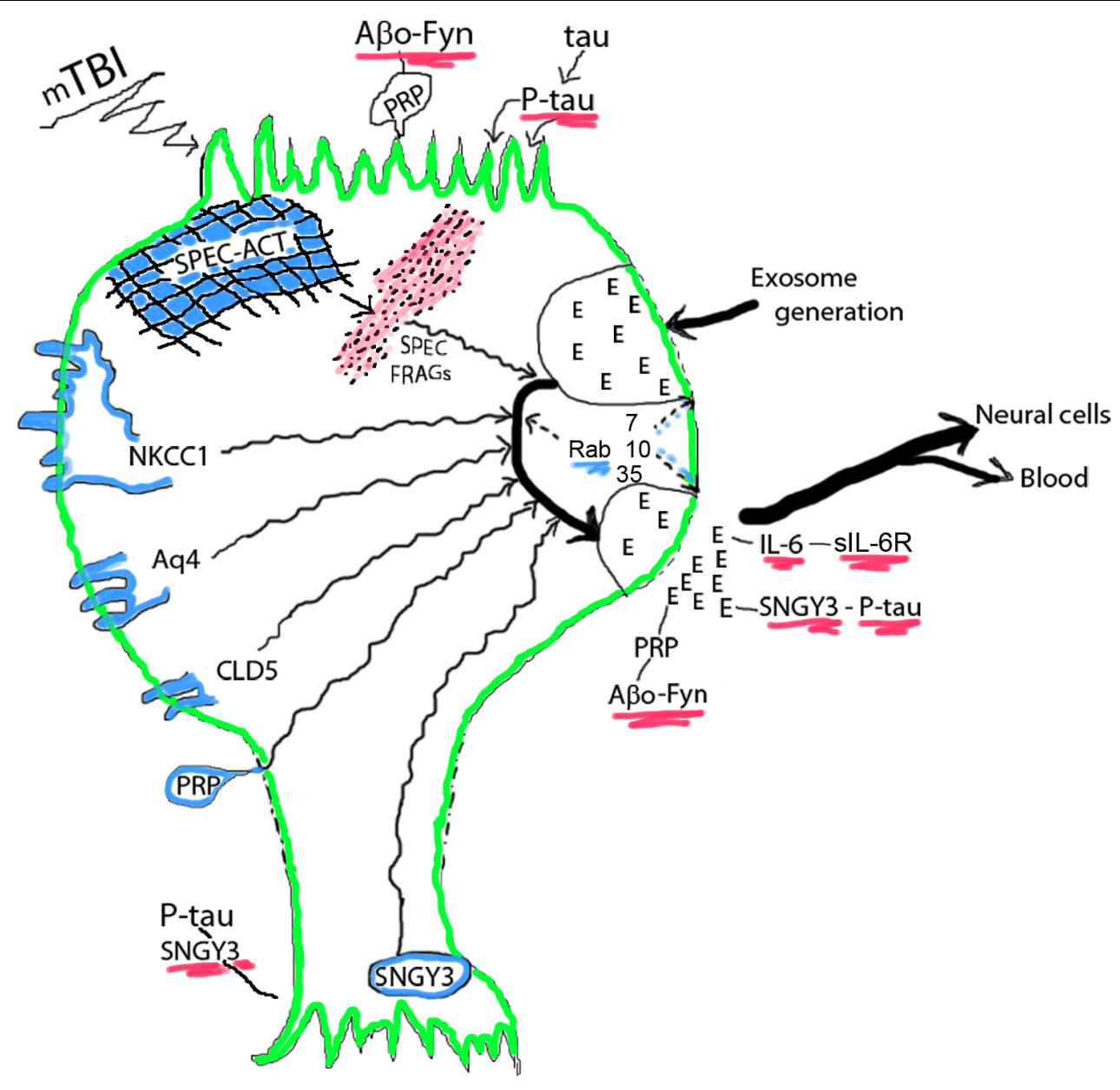

FIGURE 1 | Acute mTBI-evoked alterations in NDEs. NDEs are generated at the plasma membrane surface of the cell body, loaded with diverse neuronal proteins in their endosomal course in multivesicular bodies, and secreted through secretory type multivesicular bodies. Cargo proteins are from membrane structures or the spectrin (SPEC)-actin (ACT) matrix and their levels in NDEs increase after mTBI. The small GTPases Rab 7, 10, 35, and others regulate the processes of NDE generation, trafficking and secretion, that all diminish after mTBI reduces Rab levels. Progression factors bind, concentrate and deliver neurotoxic proteins: oligomeric A $\beta$ peptides (Aßo) by PRPc in a trimolecular complex with Fyn kinase at dendritic surfaces and in NDEs, P-tau by synaptogyrin-3 (SNGY3) at axonal and synaptic surfaces and in NDEs, and IL-6 by soluble(s)IL-6Rs. E, exosome. Color depictions are green for neuronal boundary, blue for normal cellular proteins that are found in NDE cargo and red for disrupted neuronal matrix proteins and neurotoxic progression factor complexes.

of membrane association and activity of Rab GTPases are highly dependent on controlling interactions with numerous classes of accessory proteins. In the CNS, most Rab GTPases have multiple functions. For example, Rab 7 in neurons is a critical regulator of multivesicular body maturation from early endosomes, fusion of multivesicular bodies with lysosomes to form autolysosomes, and retrograde axonal transport of vesicles with the motor protein dynein into neuron cell bodies. Similarly, Rab 35 is important for the rejuvenation of synaptic vesicles from sorting/recycling endosomes and influences neuronal actin dynamics, largely by activating Rho GTPases (Chua et al., 2010). In contrast to the increases in some neuronal Rab GTPases observed in many neurodegenerative diseases (Kiral et al., 2018), NDE levels of Rab 7, Rab 10, and Rab 35 decrease in acute mTBI. Any NDE-related functional significance of decreased levels of some Rab GTPases in acute mTBI remains to be established.

\section{POTENTIAL NDE PROTEIN PROGRESSION FACTORS IN THE CONTINUUM FROM REPETITIVE mTBIS TO CHRONIC TRAUMATIC ENCEPHALOPATHY (CTE)}

It is too early in the course of elucidating acute mTBIinitiated pathways of pathogenesis of CTE for meaningful speculation about critical mechanisms. Further, we have no data for contributions of NDE miRNAs or lipids, or of astrocyteor microglia-derived exosomes. However, a tentative analytical 
integration of existing data has identified several proteins in NDEs that may serve as factors mediating progression from repetitive mTBIs to CTE. Neuronal proteins that are elevated in NDEs acutely after mTBIs and remain significantly increased for 3 months or longer at levels higher than seen acutely include the directly neurotoxic A $\beta 42$ and P-T181-tau (Goetzl et al., 2019). Neuronal proteins that are elevated in NDEs acutely after mTBIs and remain significantly increased for 3 months or longer at levels lower than seen acutely are aquaporins, synaptogyrin-3 and XIIIa, which lack direct neurotoxicity, as well as IL-6 and PRPc, that may diminish neuronal viability (Goetzl et al., 2019). Each of these latter mTBI-generated proteins is a candidate progression factor that promotes sustained neurotoxicity indirectly through enhancement of $\mathrm{A} \beta 42$ or P-tau effects or by other mechanisms.

In the model proposed, mTBI damages and releases from neuronal membranes and other structures a wide range of functional proteins that are loaded into NDEs in endosomal pathways (Figure 1). No relationships have yet been established between the severity of a single mTBI and the magnitude or duration of increases in NDE levels of the proteins being removed by the exosomal pathway, but indirect evidence suggests that most such increases last for less than 3 months after a single mTBI. Transient decreases in NDE levels and presumptively neuronal levels of $\operatorname{Rab} 7,10$, and 35 reduce formation and secretion of NDEs for days after an acute mTBI with potential back-up of released proteins in the neurons. mTBI increases $\mathrm{NDE}$ and neuronal levels of the binding partners PRPc with $A \beta$ peptides, especially oligomeric forms of $A \beta(A \beta o)$, synaptogyrin3 with P-tau species, and IL-6 with soluble IL-6 receptors (sIL6Rs). Neurotoxic concentrations of PRPc-A $\beta$ peptide complexes at dendrites, synaptogyrin-3- P-tau complexes at synapses, and IL-6-sIL-6R complexes accumulate both in and on neurons of origin at the site of mTBI and in NDEs that transport them into neighboring neurons not damaged directly by the inciting mTBI (Figure 1). Sustained increases in aquaporins promote edema and in IL-6 maintain chronic inflammation after mTBIs. Thus acute changes of mTBI may progress to pathogenesis of early CTE with increasing probability after subsequent repetitive mTBIs (Figure 2).

The postulated stages of mTBI induction of CTE are illustrated schematically from normal to mild deterioration after one mTBI to advanced CTE pathology after repeated mTBIs (mTBIn) (Figure 2). After a single mTBI, APP and BACE1 in exosomes from reactive astrocytes $(A G)$ and to a lesser extent from damaged neurons are transferred to lesional and distant neurons. This initiates increased neuronal production of $\mathrm{A} \beta$ peptides, their

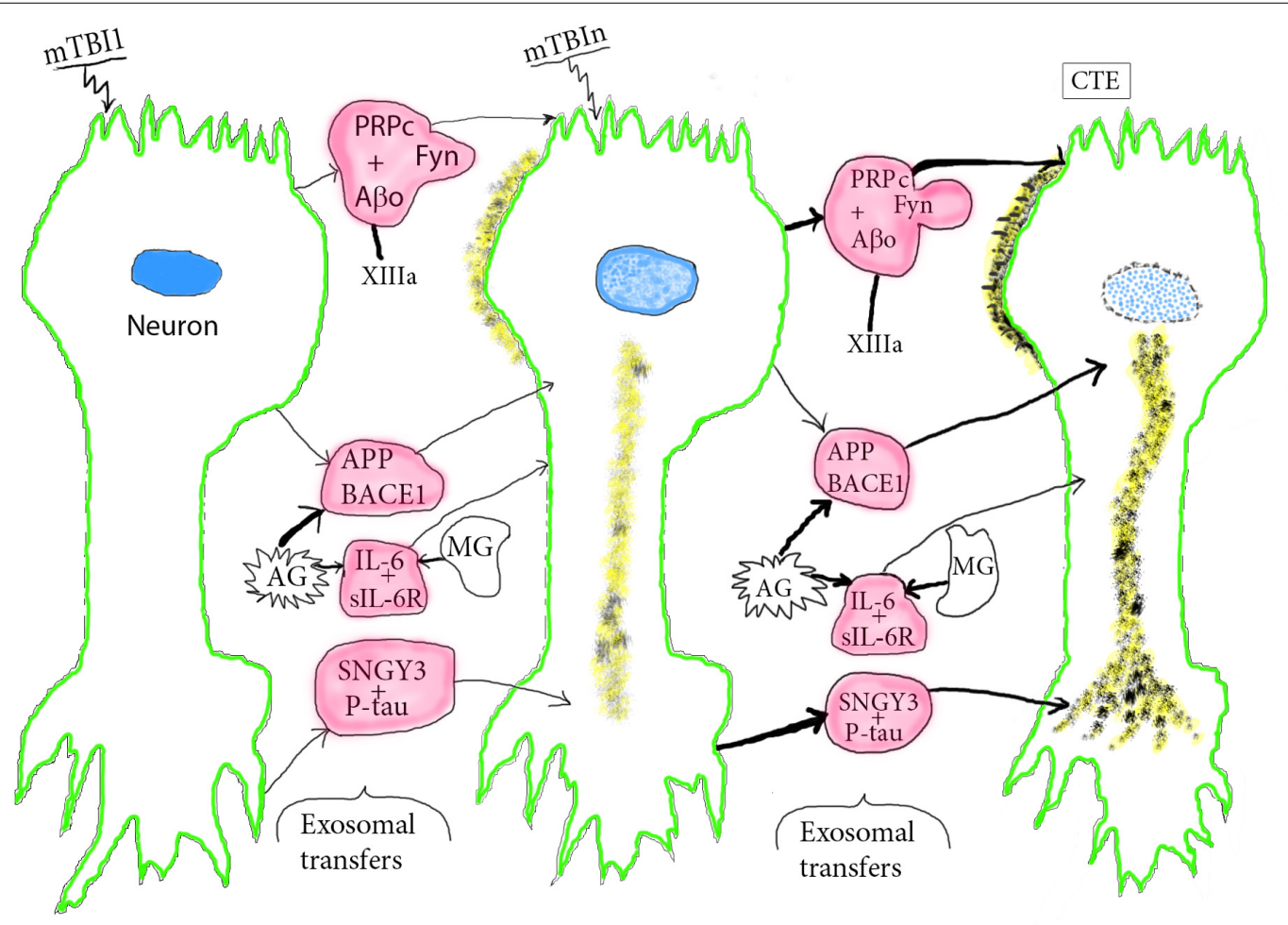

FIGURE 2 | Course of pathogenesis of CTE by repetitive mTBls. An initial mTBI leads to neuronal production of NDEs containing pathogenic complexes composed of PRPC+ABo+Fyn, SNGY3+P-tau, and IL-6-sIL-6R that begin to damage the donor neurons at sites of mTBI and other neurons that receive the neurotoxic NDEs. Microglia (MG) and astrocytes (AG) produce MDEs and ADEs, respectively, that carry increased levels of APP, BACE-1, and IL-6 capable of further neurotoxic damage of neurons that receive these other sets of exosomes. This series of processes increases with subsequent episodes of mTBI and is enhanced by other progression factors, such as XIIla that promotes oligomerization of $A \beta$ to $A \beta$ o. P-tau neuropil fibers and $A \beta$ o deposits increase in size and density with repetitive mTBls and lead to neuronal apoptosis. Color depictions are green for neuronal boundary, blue for the degenerating nucleus, yellow for A $\beta$, P-tau deposits, and red for NDEs with progression factor complexes and AG/MG products. 
oligomerization to $\mathrm{A} \beta \mathrm{o}$ that is catalyzed in part by increased levels of XIIIa (Hur et al., 2019), and beginning aggregation into loose deposits on neurons (Figure 2, second stage). IL-6sIL-6R complexes in exosomes from reactive AG and microglia $(\mathrm{MG})$ are secreted in part in their respective ADEs and MDEs for transfer to neurons, which mediates a sustained neuronal inflammatory response. PRPc-Fyn kinase-A $\beta$ peptide complexes with XIIIa-oligomerized $\mathrm{A} \beta$ ( $\mathrm{A} \beta \mathrm{O})$ are transferred by NDEs to dendrites of other neurons and synaptogyrin-3 (SNGY-3)P-tau complexes are transferred by NDEs and concentrated in axonal and presynaptic areas of other neurons to begin formation of loose P-tau strands and tangles. Repetitive mTBIs result in enhancement of all of these exosomal transfer processes with greater neuronal proteinopathic deposition of $A \beta o$ and forms of P-tau followed by early neuronal apoptosis in stage 3 (Figure 2). In summary, mTBI-derived CTE progression factors such as PRPc, XIIIa and SNGY-3, may act by binding to, concentrating and delivering to neurons neurotoxic $A \beta$ peptides and various forms of P-tau. Others act independently of neurotoxic mediators, as exemplified by IL-6, which is bound by sIL-6Rs that dominate in neural tissues, link as the IL-6sIL-6R complex to glycoprotein 130 on neurons and transmit neurodegenerative signals to the affected neurons (Erta et al., 2012; Rothaug et al., 2016). Accessory ligands for aquaporins have not been identified.

The many means for validation of the progression factor hypothesis of mediation of CTE by repetitive mTBIs include simple definitions of the time-courses of changes in NDE levels of three classes of relevant biomarkers. First, NDE levels of acute biomarkers, such as annexin VII, claudin-5, UCH-L1, spectrin fragments and NKCC-1, should only be elevated in the several weeks after each mTBI. Second, acute NDE increases in levels of $\mathrm{A} \beta$ peptide and P-tau mediators of proteinopathic toxicity should increase progressively and persist longer after each episode of mTBI. Third, NDE levels of the progression factors XIIIa, PRPc, SNGY-3, and possibly IL-6 and aquaporins should remain on elevated plateaus for months to years between episodes of mTBI. It also remains to be established if there are any relationships between the severity of a single mTBI or the stage of advancement to CTE and the NDE level of any of the biomarkers. Changes in the NDE level and duration of elevation of some biomarkers of mTBI also hopefully may provide information about the effectiveness of a therapeutic agent or program. Finally, this model may encourage extending drug discovery for mTBI and possibly Alzheimer disease to agents

\section{REFERENCES}

Agoston, D. V., Shutes-David, A., and Peskind, E. R. (2017). Biofluid biomarkers of traumatic brain injury. Brain Inj. 31, 1195-1203. doi: 10.1080/02699052.2017. 1357836

Barnes, D. E., Byers, A. L., Gardner, R. C., Seal, K. H., Boscardin, W. J., and Yaffe, K. (2018). Association of mild traumatic brain injury with and without loss of consciousness with dementia in US military veterans. JAMA Neurol. 75, 1055-1061. doi: 10.1001/jamaneurol.2018.0815

Barnes, D. E., Kaup, A., Kirby, K. A., Byers, A. L., Diaz-Arrastia, R., and Yaffe, K. (2014). Traumatic brain injury and risk of dementia in older veterans. Neurology 83, 312-319. doi: 10.1212/WNL.0000000000000616 that alter neuronal expression of progression factors and their binding of $A \beta$ peptides and P-tau species.

\section{DATA AVAILABILITY}

The datasets for this manuscript are not publicly available because data are available in Goetzl et al. (2019) cited reference. Requests to access the datasets should be directed to edward.goetzl@ucsf.edu.

\section{ETHICS STATEMENT}

This study was carried out in accordance with the recommendations of the University of California, San Francisco, San Francisco, CA, United States and University of Denver Human Research Institutional Review Committees with written informed consent from all subjects. All subjects gave written informed consent in accordance with the Declaration of Helsinki. The protocol was approved by the University of California, San Francisco and University of Denver Human Research Institutional Review Committees.

\section{AUTHOR CONTRIBUTIONS}

EG and DK formulated the conceptual framework of this perspective and prepared the draft manuscript. All authors analyzed the findings reported, edited the manuscript, and approved the final version submitted.

\section{FUNDING}

The research described was supported by a pilot grant to Drs. Ledreux and Granholm-Bentley from the Knoebel Institute for Healthy Aging and by support of Drs. Mustapic and Kapogiannis from the Intramural Program of the National Institute on Aging of the NIH.

\section{ACKNOWLEDGMENTS}

The authors are grateful to Judith H. Goetzl for preparation of the graphic illustrations.

Blasko, I., Beer, R., Bigl, M., Apelt, J., Franz, G., Rudzki, D., et al. (2004). Experimental traumatic brain injury in rats stimulates the expression, production and activity of Alzheimer's disease beta-secretase (BACE-1). J. Neural Transm. 111, 523-536. doi: 10.1007/s00702-003-0095-6

Blyth, B. J., Farhavar, A., Gee, C., Hawthorn, B., He, H., Nayak, A., et al. (2009). Validation of serum markers for blood-brain barrier disruption in traumatic brain injury. J. Neurotrauma 26, 1497-1507. doi: 10.1089/neu.2008-0738

Broglio, S. P., Sosnoff, J. J., Shin, S., He, X., Alcaraz, C., and Zimmerman, J. (2009). Head impacts during high school football: a biomechanical assessment. J. Athl. Train 44, 342-349. doi: 10.4085/1062-6050-44.4.342

Brophy, G. M., Mondello, S., Papa, L., Robicsek, S. A., Gabrielli, A., Tepas, J., et al. (2011). Biokinetic analysis of ubiquitin C-terminal 
hydrolase-L1 (UCH-L1) in severe traumatic brain injury patient biofluids. J. Neurotrauma 28, 861-870. doi: 10.1089/neu.2010. 1564

Champion, H. R., Holcomb, J. B., and Young, L. A. (2009). Injuries from explosions: physics, biophysics, pathology, and required research focus. J. Trauma 66, 1468-1477; discussion 1477. doi: 10.1097/TA.0b013e3181a27e7f

Chua, C. E., Lim, Y. S., and Tang, B. L. (2010). Rab35-a vesicular traffic-regulating small GTPase with actin modulating roles. FEBS Lett. 584, 1-6. doi: 10.1016/j. febslet.2009.11.051

Crane, P. K., Gibbons, L. E., Dams-O’Connor, K., Trittschuh, E., Leverenz, J. B., Keene, C. D., et al. (2016). Association of traumatic brain injury with late-life neurodegenerative conditions and neuropathologic findings. JAMA Neurol. 73, 1062-1069. doi: 10.1001/jamaneurol.2016.1948

Creutz, C. E. (1992). The annexins and exocytosis. Science 258, 924-931. doi: $10.1126 /$ science. 1439804

Dailey, N. S., Smith, R., Bajaj, S., Alkozei, A., Gottschlich, M. K., Raikes, A. C., et al. (2018). Elevated aggression and reduced white matter integrity in mild traumatic brain injury: a DTI study. Front. Behav. Neurosci. 12:118. doi: 10. 3389/fnbeh.2018.00118

DeKosky, S. T., Blennow, K., Ikonomovic, M. D., and Gandy, S. (2013). Acute and chronic traumatic encephalopathies: pathogenesis and biomarkers. Nat. Rev. Neurol. 9, 192-200. doi: 10.1038/nrneurol.2013.36

Di Battista, A. P., Rhind, S. G., and Baker, A. J. (2013). Application of bloodbased biomarkers in human mild traumatic brain injury. Front. Neurol. 4:44. doi: 10.3389/fneur.2013.00044

Erta, M., Quintana, A., and Hidalgo, J. (2012). Interleukin-6, a major cytokine in the central nervous system. Int. J. Biol. Sci. 8, 1254-1266. doi: 10.7150/ijbs.4679

Faul, M., and Coronado, V. (2015). Epidemiology of traumatic brain injury. Handb. Clin. Neurol. 127, 3-13.

Fiandaca, M. S., Mapstone, M., Mahmoodi, A., Gross, T., Macciardi, F., Cheema, A. K., et al. (2018). Plasma metabolomic biomarkers accurately classify acute mild traumatic brain injury from controls. PLoS One 13:e0195318. doi: 10.1371/ journal.pone. 0195318

Gardner, R. C., Burke, J. F., Nettiksimmons, J., Kaup, A., Barnes, D. E., and Yaffe, K. (2014). Dementia risk after traumatic brain injury vs nonbrain trauma: the role of age and severity. JAMA Neurol. 71, 1490-1497. doi: 10.1001/jamaneurol. 2014.2668

Gill, J., Mustapic, M., Diaz-Arrastia, R., Lange, R., Gulyani, S., Diehl, T., et al. (2018). Higher exosomal tau, amyloid-beta 42 and IL-10 are associated with mild TBIs and chronic symptoms in military personnel. Brain Inj. 32, 12771284. doi: 10.1080/02699052.2018.1471738

Goetzl, E. J., Elahi, F. M., Mustapic, M., Kapogiannis, D., Pryhoda, M., Gilmore, A., et al. (2019). Altered levels of plasma neuron-derived exosomes and their cargo proteins characterize acute and chronic mild traumatic brain injury. FASEB J. 33, 5082-5088. doi: 10.1096/fj.201802319R

Goetzl, L., Merabova, N., Darbinian, N., Martirosyan, D., Poletto, E., Fugarolas, K., et al. (2018). Diagnostic potential of neural exosome cargo as biomarkers for acute brain injury. Ann. Clin. Transl. Neurol. 5, 4-10. doi: 10.1002/acn3.499

Gyoneva, S., and Ransohoff, R. M. (2015). Inflammatory reaction after traumatic brain injury: therapeutic potential of targeting cell-cell communication by chemokines. Trends Pharmacol. Sci. 36, 471-480. doi: 10.1016/j.tips.2015.04.003

Helmy, A., De Simoni, M. G., Guilfoyle, M. R., Carpenter, K. L., and Hutchinson, P. J. (2011). Cytokines and innate inflammation in the pathogenesis of human traumatic brain injury. Prog. Neurobiol. 95, 352-372. doi: 10.1016/j.pneurobio. 2011.09.003

Higashida, T., Kreipke, C. W., Rafols, J. A., Peng, C., Schafer, S., Schafer, P., et al. (2011). The role of hypoxia-inducible factor-1alpha, aquaporin-4, and matrix metalloproteinase- 9 in blood-brain barrier disruption and brain edema after traumatic brain injury. J. Neurosurg. 114, 92-101. doi: 10.3171/2010.6. JNS10207

Hiramoto, J. S., Fernandez, C., Gasper, W., Vartanian, S., Reilly, L., and Chuter, T. (2017). Lower extremity weakness is associated with elevated blood and cerebrospinal fluid glucose levels following multibranched endovascular aortic aneurysm repair. J. Vasc. Surg. 65, 311-317. doi: 10.1016/j.jvs.2016.08.111

Hue, C. D., Cho, F. S., Cao, S., Nicholls, R. E., Vogel Iii, E. W., and Sibindi, C. (2016). Time course and size of blood-brain barrier opening in a mouse model of blast-induced traumatic brain injury. J. Neurotrauma 33, 1202-1211. doi: 10.1089/neu.2015.4067

Hur, W. S., Mazinani, N., Lu, X. J. D., Yefet, L. S., Byrnes, J. R., Ho, L., et al. (2019). Coagulation factor XIIIa cross-links amyloid beta into dimers and oligomers and to blood proteins. J. Biol. Chem. 294, 390-396. doi: 10.1074/jbc.RA118. 005352

Kim, H. J., Tsao, J. W., and Stanfill, A. G. (2018). The current state of biomarkers of mild traumatic brain injury. JCI Insight 3:97105. doi: 10.1172/jci.insight.97105

Kiral, F. R., Kohrs, F. E., Jin, E. J., and Hiesinger, P. R. (2018). Rab GTPases and membrane trafficking in neurodegeneration. Curr. Biol. 28, R471-R486. doi: 10.1016/j.cub.2018.02.010

Kuriakose, M., Rama Rao, K. V., Younger, D., and Chandra, N. (2018). Temporal and spatial effects of blast overpressure on blood-brain barrier permeability in traumatic brain injury. Sci. Rep. 8:8681. doi: 10.1038/s41598-01826813-7

Langlois, J. A., Rutland-Brown, W., and Wald, M. M. (2006). The epidemiology and impact of traumatic brain injury: a brief overview. J. Head. Trauma Rehabil. 21, 375-378. doi: 10.1097/00001199-200609000-00001

Lozano, D., Gonzales-Portillo, G. S., Acosta, S., de la Pena, I., Tajiri, N., Kaneko, Y., et al. (2015). Neuroinflammatory responses to traumatic brain injury: etiology, clinical consequences, and therapeutic opportunities. Neuropsychiatr. Dis. Treat. 11, 97-106. doi: 10.2147/NDT.S65815

McKee, A. C., Alosco, M. L., and Huber, B. R. (2016). Repetitive head impacts and chronic traumatic encephalopathy. Neurosurg. Clin. N. Am. 27, 529-535. doi: 10.1016/j.nec.2016.05.009

Mettang, M., Reichel, S. N., Lattke, M., Palmer, A., Abaei, A., Rasche, V., et al. (2018). IKK2/NF-kappaB signaling protects neurons after traumatic brain injury. FASEB J. 32, 1916-1932. doi: 10.1096/fj.201700826R

Morita, Y., Callicott, J. H., Testa, L. R., Mighdoll, M. I., Dickinson, D., and Chen, Q. (2014). Characteristics of the cation cotransporter NKCC1 in human brain: alternate transcripts, expression in development, and potential relationships to brain function and schizophrenia. J. Neurosci. 34, 4929-4940. doi: 10.1523/ JNEUROSCI.1423-13.2014

Obermeier, B., Daneman, R., and Ransohoff, R. M. (2013). Development, maintenance and disruption of the blood-brain barrier. Nat. Med. 19, 1584 1596. doi: 10.1038/nm.3407

Ohara, O., Ohara, R., Yamakawa, H., Nakajima, D., and Nakayama, M. (1998). Characterization of a new beta-spectrin gene which is predominantly expressed in brain. Brain Res. Mol. Brain Res. 57, 181-192. doi: 10.1016/s0169-328x(98) 00068-0

Oresic, M., Posti, J. P., Kamstrup-Nielsen, M. H., Takala, R. S. K., Lingsma, H. F., and Mattila, I. (2016). Human serum metabolites associate with severity and patient outcomes in traumatic brain injury. EBioMedicine 12, 118-126. doi: 10.1016/j.ebiom.2016.07.015

Papa, L., Ramia, M. M., Edwards, D., Johnson, B. D., and Slobounov, S. M. (2015). Systematic review of clinical studies examining biomarkers of brain injury in athletes after sports-related concussion. J. Neurotrauma 32, 661-673. doi: 10.1089/neu.2014.3655

Perry, D. C., Sturm, V. E., Peterson, M. J., Pieper, C. F., Bullock, T., and Boeve, B. F. (2016). Association of traumatic brain injury with subsequent neurological and psychiatric disease: a meta-analysis. J. Neurosurg. 124, 511-526. doi: 10.3171/ 2015.2.JNS14503

Prince, C., and Bruhns, M. E. (2017). Evaluation and treatment of mild traumatic brain injury: the role of neuropsychology. Brain Sci. 7:E105.

Quillinan, N., Herson, P. S., and Traystman, R. J. (2016). Neuropathophysiology of brain injury. Anesthesiol. Clin. 34, 453-464. doi: 10.1016/j.anclin.2016.04.011

Roozenbeek, B., Maas, A. I., and Menon, D. K. (2013). Changing patterns in the epidemiology of traumatic brain injury. Nat. Rev. Neurol. 9, 231-236. doi: 10.1038/nrneurol.2013.22

Roselli, F., Chandrasekar, A., and Morganti-Kossmann, M. C. (2018). Interferons in traumatic brain and spinal cord injury: current evidence for translational application. Front. Neurol. 9:458. doi: 10.3389/fneur.2018.00458

Rothaug, M., Becker-Pauly, C., and Rose-John, S. (2016). The role of interleukin6 signaling in nervous tissue. Biochim. Biophys. Acta $1863(6 \mathrm{Pt} \mathrm{A}), 1218-1227$. doi: 10.1016/j.bbamcr.2016.03.018

Roy, D., Vaishnavi, S., Han, D., and Rao, V. (2017). Correlates and prevalence of aggression at six months and one year after first-time traumatic brain injury. 
J. Neuropsychiatry Clin. Neurosci. 29, 334-342. doi: 10.1176/appi.neuropsych. 16050088

Scharfman, H. E., and Binder, D. K. (2013). Aquaporin-4 water channels and synaptic plasticity in the hippocampus. Neurochem. Int. 63, 702-711. doi: 10. 1016/j.neuint.2013.05.003

Simon, D. W., McGeachy, M. J., Bayir, H., Clark, R. S. B., Loane, D. J., and Kochanek, P. M. (2017). The far-reaching scope of neuroinflammation after traumatic brain injury. Nat. Rev. Neurol. 13:572. doi: 10.1038/nrneurol.2017.116

Stern, R. A., Tripodis, Y., Baugh, C. M., Fritts, N. G., Martin, B. M., Chaisson, C., et al. (2016). Preliminary study of plasma exosomal Tau as a potential biomarker for chronic traumatic encephalopathy. J. Alzheimers Dis. 51, 1099-1109. doi: $10.3233 /$ JAD- 151028

Taylor, C. A., Bell, J. M., Breiding, M. J., and Xu, L. (2017). Traumatic brain injury-related emergency department visits, hospitalizations, and deaths United States, 2007 and 2013. MMWR Surveill. Summ. 66, 1-16. doi: 10.15585/ mmwr.ss6609a1

Werhane, M. L., Evangelista, N. D., Clark, A. L., Sorg, S. F., Bangen, K. J., Tran, M., et al. (2017). Pathological vascular and inflammatory biomarkers of acute- and chronic-phase traumatic brain injury. Concussion 2:CNC30. doi: 10.2217/cnc2016-0022

Wilk, J. E., Herrell, R. K., Wynn, G. H., Riviere, L. A., and Hoge, C. W. (2012). Mild traumatic brain injury (concussion), posttraumatic stress disorder, and depression in U.S. soldiers involved in combat deployments: association with postdeployment symptoms. Psychosom. Med. 74, 249-257. doi: 10.1097/PSY.0b013e318244c604

Woodcock, T., and Morganti-Kossmann, M. C. (2013). The role of markers of inflammation in traumatic brain injury. Front. Neurol. 4:18. doi: 10.3389/fneur. 2013.00018

Yan, E. B., Satgunaseelan, L., Paul, E., Bye, N., Nguyen, P., Agyapomaa, D., et al. (2014). Post-traumatic hypoxia is associated with prolonged cerebral cytokine production, higher serum biomarker levels, and poor outcome in patients with severe traumatic brain injury. J. Neurotrauma 31, 618-629. doi: 10.1089/neu. 2013.3087

Conflict of Interest Statement: The authors declare that the research was conducted in the absence of any commercial or financial relationships that could be construed as a potential conflict of interest.

The handling Editor declared a past co-authorship with one of the authors DK.

Copyright (c) 2019 Goetzl, Ledreux, Granholm, Elahi, Goetzl, Hiramoto and Kapogiannis. This is an open-access article distributed under the terms of the Creative Commons Attribution License (CC BY). The use, distribution or reproduction in other forums is permitted, provided the original author(s) and the copyright owner(s) are credited and that the original publication in this journal is cited, in accordance with accepted academic practice. No use, distribution or reproduction is permitted which does not comply with these terms. 